\title{
Left-handed metamaterial coatings for subwavelength-resolution imaging
}

\author{
Carlos J. Zapata-Rodríguez, ${ }^{1, *}$ David Pastor, ${ }^{1}$ Luis E. Martínez, ${ }^{2}$ and Juan J. Miret ${ }^{2}$ \\ ${ }^{1}$ Department of Optics, University of Valencia, Dr. Moliner 50, 46100 Burjassot, Spain \\ ${ }^{2}$ Department of Optics, Pharmacology and Anatomy, University of Alicante, P.O. Box 99, Alicante, Spain \\ ${ }^{*}$ Corresponding author: carlos.zapata@uv.es
}

Received May 7, 2012; revised July 10, 2012; accepted July 28, 2012;

posted July 30, 2012 (Doc. ID 168038); published August 30, 2012

\begin{abstract}
We report on a procedure to improve the resolution of far-field imaging by using a neighboring high-index medium that is coated with a left-handed metamaterial. The resulting plot can also exhibit an enhanced transmission by considering proper conditions to retract backscattering. Based on negative refraction, geometrical aberrations are considered in detail since they may cause a great impact in this sort of diffraction-unlimited imaging by reducing its resolution power. We employ a standard aberration analysis to refine the asymmetric configuration of metamaterial superlenses. We demonstrate that low-order centrosymmetric aberrations can be fully corrected for a given object plane. For subwavelength-resolution imaging, however, high-order aberrations become of relevance, which may be balanced with defocus. Not only the point spread function but also numerical simulations based on the finite-element method support our theoretical analysis, and subwavelength resolution is verified in the image plane. (C) 2012 Optical Society of America OCIS codes: $\quad 110.2990,160.3918,220.1000$.
\end{abstract}

\section{INTRODUCTION}

An asymmetric flat superlens is a film made of a left-handed metamaterial (LHM) that is deposited on a smooth, transparent body such as glass with positive dielectric constant. Therefore the object space has an index of refraction (IR) different from that in the image space, leading to an asymmetric arrangement. Originally, this idea was conceived because using a solid substrate, this imaging device will be mechanically much more stable than a layer sustained in free space [1]. There, only asymmetric silver superlenses were analyzed in detail, for which amplification of evanescent waves sustained by surface waves is more favorable if the real part of the dielectric constant of the metal and the substrate matches except for its sign.

In the case that the lensing flat slab shows effectively a negative permeability, negative refraction allows imaging mainly using homogeneous waves. Moreover, if the IR of the output medium is higher than that IR corresponding to the medium surrounding the object, some evanescent waves emitted by the source become homogeneous after passing through the lens. This fact allows the formation of far-field images with subwavelength resolution.

Unfortunately there is no perfect image plane in the asymmetric arrangement and the image suffers from aberrations. Note that the root of aberrations in symmetric superlenses is diverse and may be caused by materials having an impedance (and IR) not matched to free space [2, 2,3$]$, material losses $[4,5]$, because the equifrequency curve is slightly deformed from an ideal spherical shape particularly for large angular components [6,7], and caused by the anisotropic effect from nonmagnetic anisotropic media [8,9]. Here we focus on the IR mismatching that comes naturally in the asymmetric configuration. Moreover, Seidel aberrations have been discussed in different kinds of imaging nanostructures like metallodielectric photonic crystals (PCs) [10,11], negative-refractive lenses fabricated out of a silicon-on-insulator PC slab [12], graded PC lenses [13], and spherical lenses composed of LHMs $[\underline{14}, \underline{15}]$. In the previous examples, nonapertured superlenses are usually considered and oblique aberrations may be disregarded. Finally, in order to avoid this awkward situation, a transformation design of optical elements that perform imaging has been proposed, free from geometric aberrations [16], though it seems far from being experimentally demonstrated.

In this paper we investigate the effects of primary spherical aberration (SA) and higher-order SA in LHM asymmetric lenses. In Section 2, primary SA is corrected for a given object plane; however, some residual aberrations for nearby object planes remain. This effect is not observed in perfect symmetric lenses since it is caused by the breaking of the shift invariance along the optic axis driven by asymmetry of this new imaging problem. In Section 3 we show that the unbalanced arrangement allows proper conditions to retract backscattering. The diffractive behavior of SA-corrected metamaterial coatings are disclosed in Section $\underline{4}$ by using electric dipole fields. Here we demonstrate subwavelength capabilities in far-field imaging. In Section $\underline{5}$ we provide the point spread function (PSF) of such antireflection superlenses and we estimate the limit of resolution unambiguously. Finally, the main conclusions are outlined in Section 6 .

\section{SPHERICAL ABERRATION IN FLAT LENSES}

Let us consider the asymmetric flat lens shown in Fig. 1. A point object $O_{1}$ is suspended at a distance $s_{1}$ from the front face of the superlens made of a material exhibiting negative IR, $n_{2}<0$. Assuming that the object space is characterized by 


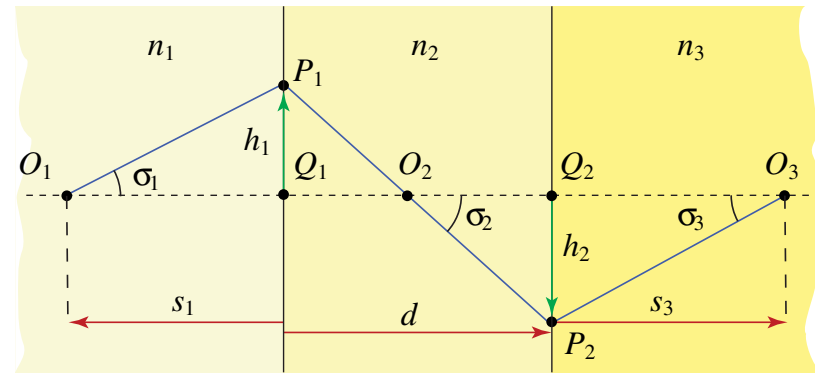

Fig. 1. (Color online) Schematic representation of an asymmetric flat lens of refractive index $n_{2}$ and width $d$.

an IR $n_{1}>0$, and that the width $d$ of the lens is sufficiently large, a real Gaussian image $O_{2}$ is formed inside the LHM [17]. Traveling through the lens exit surface we reproduce the secondary, outlying image at $O_{3}$ in a semi-infinite dielectric of IR $n_{3}$. Provided the IR in the image space turns out to be positive, $n_{3}>0$, the image point $O_{3}$ is also real, which is located at a distance $s_{3}$. Note that we employ oriented axial distances, i.e., $s_{1}<0$ and $s_{3}>0$ for a real object and a real image, respectively. Also the lens width $d>0$.

For a nonapertured setup, the chief ray joins the points $O_{1}$, $\mathrm{O}_{2}$ and the Gaussian image $\mathrm{O}_{3}$ by means of the same straight line. Let us evaluate the aberration of a ray passing through the point $P_{2}$, which is placed on the exit surface at a height $h_{2}$, with respect to the chief ray. This aberration is estimated by the optical-path difference of both light rays, i.e., $W=$ $n_{1}\left(\overline{O_{1} P_{1}}-\overline{O_{1} Q_{1}}\right)+n_{2}\left(\overline{P_{1} P_{2}}-\overline{Q_{1} Q_{2}}\right)+n_{3}\left(\overline{P_{2} O_{3}}-\overline{Q_{2} O_{3}}\right)$. Note that $\overline{O_{1} Q_{1}}=-s_{1}, \overline{Q_{1} Q_{2}}=d$, and $\overline{Q_{2} O_{3}}=s_{3}$. Finally, this ray aberration reads approximately [18]

$$
W\left(h_{2}\right) \approx{ }_{0} a_{20} h_{2}^{2}+{ }_{0} a_{40} h_{2}^{4}+{ }_{0} a_{60} h_{2}^{6} .
$$

The aberration terms ${ }_{0} a_{20},{ }_{0} a_{40}$, and ${ }_{0} a_{60}$ are attributed to defocus, primary SA, and fifth-order SA, respectively. These aberration coefficients are evaluated by using the geometrical relations $\tan \sigma_{1}=h_{1} / s_{1}$ and $\tan \sigma_{2}=\left(h_{1}-h_{2}\right) / d$, and the Snell law

$$
n_{1} \sin \sigma_{1}=n_{2} \sin \sigma_{2}
$$

In particular, the Gaussian image plane is given under the condition ${ }_{0} a_{20}=0$, which yields

$$
s_{3}=n_{3}\left(\frac{s_{1}}{n_{1}}-\frac{d}{n_{2}}\right)
$$

Therefore an axial displacement of the object point $O_{1}$ changing $s_{1}$ leads to an image shift following a direct proportion, as shown in Fig. 2(a). Note that a real paraxial image $O_{3}$ is attained with the condition $d \geq s_{1} n_{2} / n_{1}$, that is, if the secondary paraxial image $\mathrm{O}_{2}$ is also a real image.

At the Gaussian image point $O_{3}$, where Eq. (3) is satisfied, the aberration coefficient for primary SA gives

$$
{ }_{0} a_{40}=\frac{n_{1} n_{2}\left[n_{1}^{3}\left(n_{2}^{2}-n_{3}^{2}\right) d+n_{2}^{3}\left(n_{3}^{2}-n_{1}^{2}\right) s_{1}\right]}{8 n_{3}^{2}\left(n_{1} d-n_{2} s_{1}\right)^{4}} .
$$

Equation (4) is obtained from Eq. (1) by using a Taylor expansion of $W$ around $h_{2}=0$. This result is slightly different if the aberration is calculated surface by surface, and the aberration

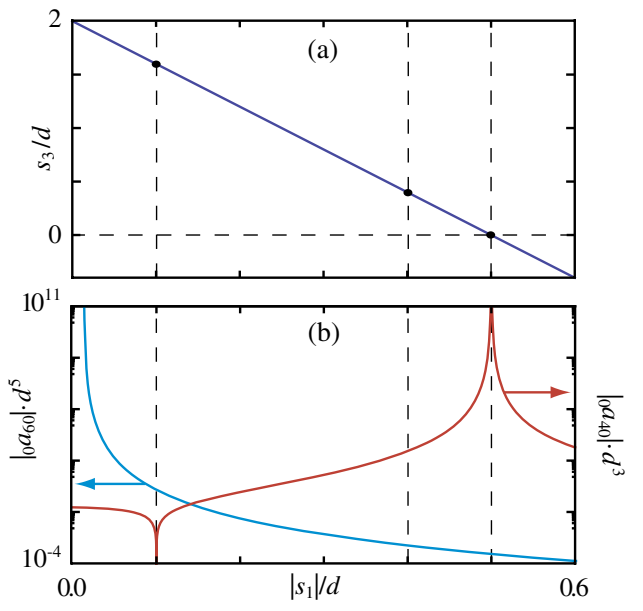

Fig. 2. (Color online) Geometrical imaging for a flat lens of $n_{2}=-2$ sandwiched between dielectric media of indices of refraction $n_{1}=1$ and $n_{3}=4$. (a) Gaussian imaging based on Eq. (3). (b) Red line represents primary SA given by Eq. ( $\underline{4}$ ) and blue line represents fifth-order SA given by Eq (6).

of the flat lens is obtained by adding the aberration contributions of all its surfaces [18]. Note that primary SA cannot be totally corrected for $0 \leq-s_{1}<\infty$ when $n_{1}=n_{3}$ except for the perfect lens, where additionally $n_{2}=-n_{3}$. This is a wellknown case where high-order aberration coefficients also vanish leading to stigmatic imaging. Also a plane-parallel asymmetric plate may be corrected of primary SA. Provided the equation ${ }_{0} a_{40}=0$ is satisfied, we obtain a linear relationship between the lens width $d$ and the on-axis object distance

$$
s_{1}=\frac{n_{1}^{3}\left(n_{3}^{2}-n_{2}^{2}\right)}{n_{2}^{3}\left(n_{3}^{2}-n_{1}^{2}\right)} d
$$

in terms of the IRs of the media involved. A given flat lens cannot be corrected of primary SA for more than one object plane, as shown in Fig. 2(b), and therefore images originated from scatterers that fail to keep Eq. (5) suffer from SA. Furthermore, the primary SA coefficient ${ }_{0} a_{40}$ diverges for the limiting case $s_{3}=0$, excepting when $n_{1}=-n_{2}$, leading to perfect geometric imaging. Therefore quality of the (real) image improves as the (real) object point $O_{1}$ come close to the input surface.

In Fig. 3 , we plot a ray tracing for a flat metamaterial lens of $n_{2}=-2$ surrounded by object and image media of IRs $n_{1}=1$ and $n_{3}=4$. Fixing the lens width $d$, Eqs. (3) and (5) provide the values $s_{3}=1.6 d$ and $s_{1}=-0.1 d$, respectively. The corresponding ray tracing is shown in Fig. 3(a). Stigmatic imaging may produce a convergent focused beam of numerical aperture $n_{3} \sin \alpha=n_{1}$ that leads to an angular semiaperture $\alpha=14.5^{\circ}$. In our case, the numerical aperture is slightly reduced down to an effective value $\alpha_{\text {eff }}=10.9^{\circ}$ caused by noncorrected high-order aberrations. To inspect the deterioration of the image due to SA effects, we also present in Fig. 3(b) the ray tracing for a point object placed at $s_{1}=-0.4 d$ further from the lens entrance surface. We observe a ray distribution that is barely confined around the Gaussian image point $\mathrm{O}_{3}$, represented as a green dot in the image space. As a limiting case, we plot in Fig. 3(c) the trajectories of rays emerging from a point that is located at $s_{1}=-0.5 d$ that leads to $\left.\right|_{0} a_{40} \mid \rightarrow \infty$.

We point out that Eq. (5) gives a negative value of $s_{1}$ provided that the IR $n_{3}$ in the image plane is either higher 


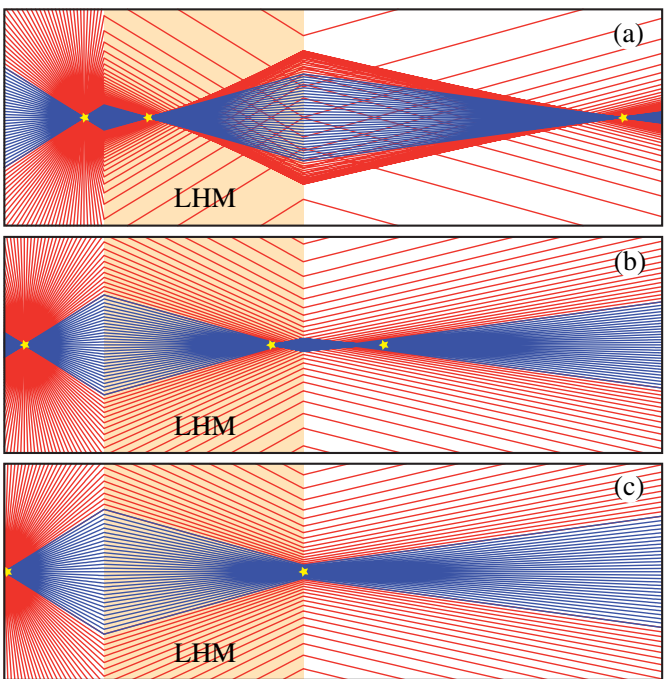

Fig. 3. (Color online) Ray tracing for an object point located at (a) $s_{1}=-0.1 d$, which is corrected of primary SA, (b) $s_{1}=-0.4 d$, and (c) $s_{1}=-0.5 d$ from the front surface of a flat lens of width $d$. Indices of refraction are the same as in Fig. 2. Traces corresponding to paraxial (slope lower than $30^{\circ}$ ) and nonparaxial rays are drawn in different colors. Small light-colored stars represent conjugated points.

or lower than $n_{1}$ and $\left|n_{2}\right|$ simultaneously. In order to achieve a subwavelength effect, we aim for transforming evanescent waves emitted by the source $O_{1}$ into homogeneous wave modes in the image space. In this case it is preferable for a high-index transparent medium $n_{3}>n_{1}$ to register the image.

The aberration coefficient ${ }_{0} \alpha_{60}$ for the fifth-order SA may be estimated analytically. Provided Eqs. (3) and (5) are satisfied, this high-order SA coefficient gives

$$
{ }_{0} a_{60}=-\frac{n_{1}^{13}\left(n_{1}^{2}-n_{3}^{2}\right)\left(n_{2}^{2}-n_{3}^{2}\right)^{6}}{16 n_{2}^{2} n_{3}^{14}\left(n_{2}^{2}-n_{1}^{2}\right)^{5} s_{1}^{5}} \text {. }
$$

Therefore, assuming $n_{1} \neq n_{3},{ }_{0} a_{60}$ vanishes only for the following trivial solutions: (1) imaging under mirror-symmetry negative refraction on the output surface when $n_{3}=-n_{2}$ and $s_{1}=0$, and (2) incidence of a collimated bundle of rays for which $s_{1}$ tends to infinity. Excepting these special cases, fifth-order SA cannot be corrected. In Fig. 2(b) we present ${ }_{0} a_{60} \mid$ for a given numerical example.

To conclude this section, let us make an explanatory remark concerning the IRs of the media involved on the analysis of the image formation. The ratio $n_{3} / n_{1}-1$ provides the relative enlargement of spatial bandwidth corresponding to evanescent waves in the object space that are transformed into homogeneous plane waves in the image space. This is clearly a subwavelength effect, which has been exploited elsewhere [19]. In image formation, this physical phenomenon leads to a superresolving effect, which will be developed in Section 5 . On the other hand, we point out that the value of $n_{2}$ is arbitrarily chosen provided it takes a negative value. In fact, this is a degree of freedom that may be profited at the time of imposing an additional constraint of interest.

\section{CONTROL OF REFLECTION LOSSES}

In order to take a suitable choice for the value of $n_{2}$, we considered the reflection and transmission properties of light that impinges obliquely onto the LHM thin film, which has been deposited on top of the transparent substrate. The coefficient of reflection $(r)$ and the coefficient of transmission $(t)$ evaluated from the object plane in front of the asymmetric layered lens to the image plane are [20]

$$
\begin{aligned}
& r=\frac{r_{1,2}+r_{2,3} \exp \left(2 i \beta_{2} d\right)}{1+r_{1,2} r_{2,3} \exp \left(2 i \beta_{2} d\right)} \exp \left(-2 i \beta_{1} s_{1}\right), \\
& t=\frac{t_{1,2} t_{2,3} \exp \left(-i \beta_{1} s_{1}+i \beta_{2} d+i \beta_{3} s_{3}\right)}{1+r_{1,2} r_{2,3} \exp \left(2 i \beta_{2} d\right)}
\end{aligned}
$$

Here the propagation constant is

$$
\beta_{i}=\sigma_{i} \sqrt{\varepsilon_{i} \mu_{i} k_{0}^{2}-\vec{k}_{\perp} \cdot \vec{k}_{\perp}}
$$

where $\sigma_{i}=1$ for the dielectrics and $\sigma_{2}=-1$ for the LHM, $k_{0}=$ $2 \pi / \lambda_{0}$ is the wavenumber in vacuum, and $\varepsilon_{i}$ and $\mu_{i}$ stand for the relative permittivity and permeability of the media involved, respectively. Also $\vec{k}_{\perp}=\left(k_{x}, k_{y}\right)$ is the transverse wave vector, i.e., the projection of the wave vector of the incident field over a plane that is parallel to each flat-lens interface. The Airy's formulae (7) depend on the reflection coefficient at a single interface,

$$
r_{i, j}=\frac{\mu_{j} \beta_{i}-\mu_{i} \beta_{j}}{\mu_{j} \beta_{i}+\mu_{i} \beta_{j}},
$$

which is valid for $s$-polarized waves. For $p$-polarized waves, $r_{i, j}=\left(\varepsilon_{j} \beta_{i}-\varepsilon_{i} \beta_{j}\right) /\left(\varepsilon_{j} \beta_{i}+\varepsilon_{i} \beta_{j}\right)$ applies directly to the transverse magnetic field. In all cases, they also depend on the transmission coefficient $t_{i, j}=1+r_{i j}$.

A LHM layer with

$$
\beta_{2} d=-(2 m+1) \pi / 2
$$

for $m=0,1,2 \ldots$ can be used to eliminate the reflection of light completely, which is intrinsically a dispersive phenomenon depending upon $k_{0}$. This is commonly denominated an antireflecting coating. For that purpose we additionally impose $r_{1,2}=r_{2,3}$ in Eq. ( $\underline{7 \mathrm{a}}$ ), leading to $r=0$. This condition is satisfied if

$$
\beta_{2}^{2} \mu_{1} \mu_{3}=\mu_{2}^{2} \beta_{1} \beta_{3},
$$

assuming that the wave fields are $s$-polarized. For normally incident light, i.e., $k_{\perp}=0$, Eq. (11) is simply $Z_{2}^{2}=Z_{1} Z_{3}$, where $Z_{i}$ is the intrinsic impedance of the medium $i$. Obviously, this equation for zero reflectance is a result that applies for all states of polarization. Moreover, assuming that $\mu_{i}=\sigma_{i}$, we finally obtain a condition $n_{2}=-\sqrt{n_{1} n_{3}}$ involving the IRs of all media. Note that the latter equation is held in simulations shown in Fig. 2 , and it is well-known in the theory of antireflecting films when its IR is $\left|n_{2}\right|$. Finally, a quarter-wave layer satisfying Eq. (10) with $d=\lambda_{2} / 4\left(+m \lambda_{2} / 2\right.$ for $\left.m \neq 0\right)$, being $\lambda_{2}=\lambda_{0} /\left(-n_{2}\right)$, is of interest.

In Figs. 4(a) -4 (c), we show the transmission coefficient $t$ that has been evaluated for $s$-polarized waves and superlenses of different widths. Optimum geometrical conditions are assumed under all circumstances, where Eqs. (3) and (5) are satisfied. We observe that $|t| \approx 0.5$ for $k_{\perp}=0$ in all cases 

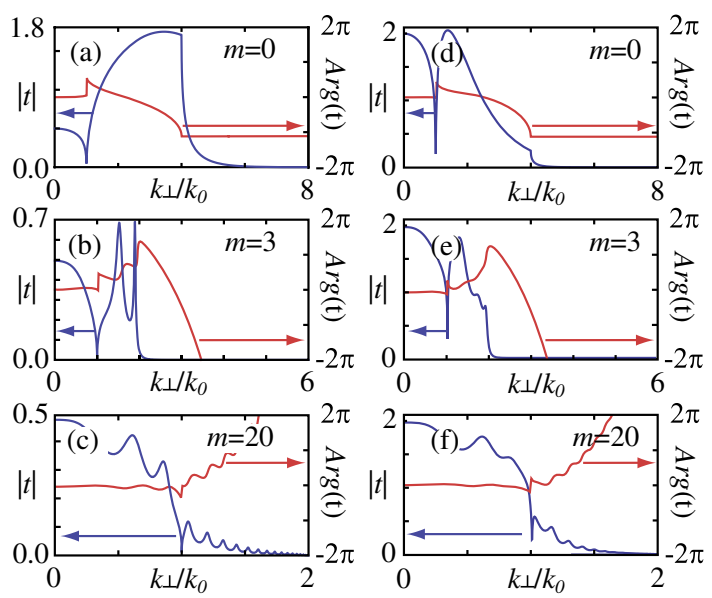

Fig. 4. (Color online) Transmission coefficient (modulus and argument) for (a)-(c) $s$-polarized waves and (d)-(f) $p$-polarized waves in a superlens of $\mu_{2}=-1+i 0.001$ and $\varepsilon_{2}=-4+i 0.001$. Surrounding transparent media have again indices of refraction $n_{1}=1$ and $n_{3}=4$. We consider different widths for the LHM flat lens: (a) and (d) $d=0.125 \lambda_{0}$; (b) and (e) $d=0.875 \lambda_{0}$; (c) and (f) $d=5.125 \lambda_{0}$. Note that all the horizontal scales are not the same.

and, therefore, reflection is extinguished (Transmittance for $s$-polarized waves is $\left.T_{s}=|t|^{2} n_{3} / n_{1}\right)$. However, reflectance might have a certain significance in higher spatial frequencies. We also observe a flat variation of the argument of $t$ for $k_{\perp}<k_{0}$, which is a consequence of eliminating primary SA. For ultrathin slabs, however, $|t|$ is of relevance at higher frequencies. By considering a boundless medium of IR $n_{1}=1$, note that there is no time-averaged power flow for $k_{\perp}>k_{0}$. The field intensity within this spectral domain, in the presence of the superlens, is by no means zero, and transmittance might reach values higher than unity, as seen for $m=0$. In these cases, the time average of the power flow in the object space is supported partially by evanescent waves that, in principle, can contribute to the far field. For them, the phase in the spatial spectrum changes by far and, therefore, aberrated images are expected in the Gaussian image plane. In Figs. 4(d)-4(f), we also present the transmission coefficient $t$ in amplitude and phase for $p$-polarized waves. Finally, from Figs. 4(a) and $4(\mathrm{~d})$ we observe that those spatial frequencies surpassing $4 k_{0}$ have a small contribution to the image formation; note that the wave field also falls off fast in the transit from the output plane of the lens toward the image plane, thus frustrating a three-dimensional (3D) focusing [21].

For a slab width much higher than the wavelength, the evanescent waves emitted by the source point $O_{1}$ cannot reach the entrance face and homogeneous waves satisfying $k_{\perp} \leq$ $k_{0}$ contribute effectively to the transmitted field in the image space, as shown in Figs. $\underline{4(\mathrm{c})}$ and $\underline{4(\mathrm{f})}$ for $d=5.125 \lambda_{0}$. Decreasing $d$ down to values close to $\lambda_{0}$ leads to the conversion of evanescent waves in the medium 1 to homogeneous waves in the medium 2. In Figs. $\underline{4(\mathrm{~b})}$ and $\underline{4(\mathrm{e})}$, we observe a critical participation of waves with transverse spatial frequencies $k_{0}<k_{\perp}<2 k_{0}$ for a lens width $d=0.875 \lambda_{0}$. In the limit $d=$ $0.125 \lambda_{0}$ associated with $m=0$, we include the spatial bandwidth into the interval $2 k_{0}<k_{\perp}<4 k_{0}$ involving evanescent waves in media 1 and 2, which are transformed into homogeneous waves in the image space, as seen in Figs. $\underline{4(\mathrm{a})}$ and $\underline{4(\mathrm{~d})}$. For that reason such a spectral stretching allows a subwavelength-resolution effect in the formation of far-field images.

\section{IMAGING ELECTRIC DIPOLE FIELDS}

It is commonly accepted that electric dipole fields, due to their high spatial confinement, are electromagnetic sources appropriate for the examination of the limit of resolution in nearfield superlenses $[22,23]$. For that purpose we use the field distribution generated by an infinite line source reducing 3D calculations to a simpler two-dimensional (2D) problem. The orientation of the line emitter lies along the $y$-axis, which is parallel to the input and output surfaces of the thin LHM coating. The electric dipole field results from elementary point dipoles with dipole moments, $p$, which are presumed to be aligned also in the $y$ direction, as shown in Fig. $\underline{5}$. In an unbounded transparent medium of permittivity $\varepsilon_{1}$ and permeability $\mu_{1}$, the electric field may be written as [24]

$$
\vec{E}=i \frac{k_{1}^{2} p}{4 \varepsilon_{1}} H_{0}^{(1)}\left(k_{1} R_{0}\right) \hat{y}
$$

where $k_{1}^{2}=k_{0}^{2} \varepsilon_{1} \mu_{1}$, the unit vector $\hat{y}$ is oriented along the $y$ axis, and $R_{0}=\sqrt{x^{2}+z^{2}}$ denotes the distance from the line source to the point under observation within the plane $x z$. Also $H_{0}^{(1)}$ is the Hankel function of the first kind, which may be written in an integral representation as

$$
H_{0}^{(1)}\left(k_{1} R_{0}\right)=\frac{1}{\pi} \int_{-\infty}^{\infty} \frac{\exp \left(i k_{x} x+i \beta_{1} z\right)}{\beta_{1}} \mathrm{~d} k_{x},
$$

for $z>0$. Note that $k_{y}=0$ here. If we consider the electric field that is transmitted through the thin LHM superlens shown in Fig. 1, assuming that the line dipole is placed at $O_{1}$, it finally yields

$$
\vec{E}=i \frac{k_{1}^{2} p}{4 \pi \varepsilon_{1}} \hat{y} \int_{-\infty}^{\infty} t\left(k_{x}\right) \frac{\exp \left(i k_{x} x+i \beta_{3} z\right)}{\beta_{1}} \mathrm{~d} k_{x} .
$$

In this equation we employ the transmission coefficient $t$ evaluated from the object plane to the image plane, which is given in Eq. (7b), for $s$-polarized waves. Therefore $z=0$ stands for the image plane.

In Fig. $\underline{6}$, we present the modulus of the electric field $|E|$ that is emitted by an electric line dipole and that is transmitted through a LHM lens of $\mu_{2}=-1+i 0.001$ and $\varepsilon_{2}=-4+i 0.001$ and different widths. The flat lens is sandwiched between media of IR $n_{1}=1$ and $n_{3}=4$. The object point $O_{1}$ is placed at a distance $s_{1}=0.1 d$ from the superlens, following Eq. (5) to minimize primary SA. We compute the scattered field within the interval $z \geq-s_{3}$ constituting the real image space. The

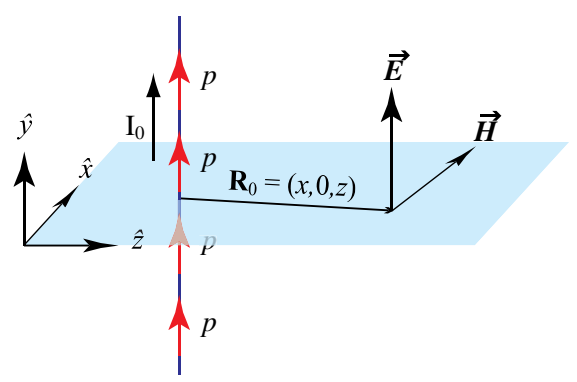

Fig. 5. (Color online) Electric line dipole composed of a continuous distribution of point dipoles that are oriented in the $y$ direction and simulating a current $I_{0}$ flowing along the $y$-axis. 
(a)

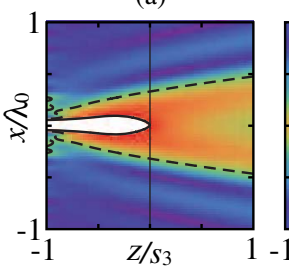

(d)

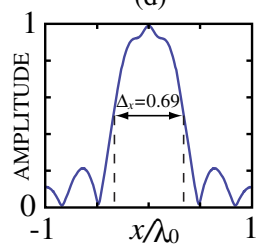

(b)

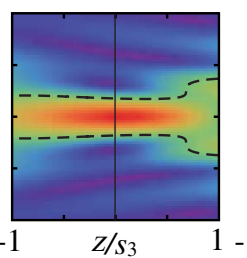

(e)

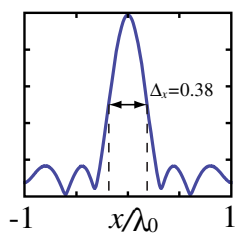

(c)

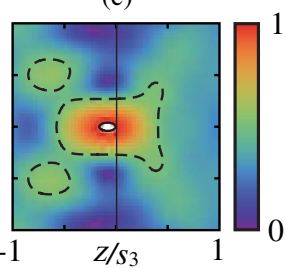

(f)

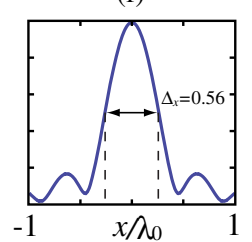

Fig. 6. (Color online) Modulus of the electric field emitted by a line electric dipole and transmitted through a negative-index slab with $\mu_{2}=-1+i 0.001$ and $\varepsilon_{2}=-4+i 0.001$ and different widths: (a) $d=0.125 \lambda_{0}$, (b) $d=0.875 \lambda_{0}$, and (c) $d=5.125 \lambda_{0}$. In all cases we present the field within $z \geq-s_{3}$. The density plots are normalized to unity at the paraxial image point $(x, z)=(0,0)$. The dashed line indicates points where amplitude falls off $1 / 2$. The thin vertical line marks the Gaussian image plane. The wave fields corresponding to the Gaussian image plane for (a), (b), and (c) are plotted in (d), (e), and (f), respectively.

numerical simulations were performed using a finite-element method and also employing Eq. (14) to verify the validity of our results.

If the superlens has a width $d$ below the wavelength, as used in Fig. 6(a), one would expect to achieve superresolution. In this case, however, the FWHM of the modulus of the electric field in the Gaussian image plane yields $\Delta_{x}=$ 0.69 in units of $\lambda_{0}$, even exceeding the limit of resolution applied to diffraction-limited systems. In order to understand such a behavior, we analyze the transmission coefficients plotted in Fig. 3(a). We observe that the phase is stabilized for $\left|k_{x}\right|<k_{0}$, but it has a fast decreasing variation for higher spatial frequencies. In this sense, the effective bandwidth in the transmission coefficient for frequencies associated with inhomogeneous waves in the medium 1 is, in practical terms, three times larger than the bandwidth for homogeneous waves; therefore the unbalanced contribution of the different spatial frequencies will make the image recovery difficult. A simple defocus provoked by a shift of the image plane toward the LHM lens serves to diminish the phase variation and aberration effects in the image. Exactly at the exit surface of the superlens, the FWHM of the electric field is $\Delta_{x}=0.086$, leading to a subwavelength resolution.

A different behavior is expected for $d \approx \lambda_{0}$. Figure $\underline{6(\mathrm{~b})}$ illustrates the diffraction behavior of a thin LHM film in the case that $d=0.875 \lambda_{0}$; that is, $m=3$ in Eq. (10). The limit of resolution has decreased substantially in the Gaussian image plane, where $\Delta_{x}=0.38$. Taking in mind the results shown in Fig. 3(b), this superresolving response is attributed to evanescent waves in medium 1 that are converted into homogeneous waves in medium 2 , which belong to the spectral range $k_{0}<\left|k_{x}\right|<2 k_{0}$. In this spectral band, however, the coefficient of transmission presents some strong variations in its phase, preventing us from the observation of an aberration-free image. Moreover, the phase of the transmission coefficient increases with $k_{x}$ so that a defocus is expected to balance high-frequency aberrations. Contrarily to the previous case, the on-axis shift must be performed moving far from the LHM lens in order to achieve the minimum spot size. In practice, the resolution improvement that is attainable with defocus may be considered negligible.

Finally, if $d=5.125 \lambda_{0}$, as shown in Fig. 6(c), the FWHM of the central lobe in the paraxial image plane yields $\Delta_{x}=0.56$ in units of $\lambda_{0}$, which is close to the diffraction limit, $\lambda_{0} / 2$. In this case, the depth of focus is significantly short, which allows the evaluation of the FWHM along the $z$-axis. This gives $\Delta_{z}=9.81$, also in units of $\lambda_{0}$. We conclude that the focused wave field is localized much stronger in the transverse direction than on axis.

The analysis that we have carried through in Section 4 so far is essentially for $s$-polarization. We point out that a similar $p$-polarization analysis is also possible based on magnetic dipoles aligned along the $y$-axis. For that purpose, now we consider a line source with a uniform distribution of elementary magnetic dipole moment $m$, which is placed at $O_{1}$, and for which the magnetic field in the image space is simply

$$
\vec{H}=i \frac{k_{1}^{2} m}{4 \pi \mu_{1}} \hat{y} \int_{-\infty}^{\infty} t\left(k_{x}\right) \frac{\exp \left(i k_{x} x+i \beta_{3} z\right)}{\beta_{1}} \mathrm{~d} k_{x} .
$$

Making use of the duality theorem [24], here $t$ from Eq. (7b) is evaluated for $p$-polarized waves. Again $z=0$ stands for the image plane. In Fig. 7, we present the modulus of the magnetic field as it is transmitted through the same LHM lens of $\mu_{2}=$ $-1+i 0.001$ and $\varepsilon_{2}=-4+i 0.001$. By comparing the wave field in Fig. 7 with the scattered field represented in Fig. 6 , for different widths of the LHM slab, we conclude that discrepancies are appreciable, in general, which are clearly attributed to departures in the coefficient of transmission $t$ for both polarizations. Also we find that the spot size of the wave field is lower for magnetic dipoles. For convenience, let us leave in Section 5 the discussion concerning how polarization impacts upon the limit of resolution of LHM coatings.

\section{PSF AND LIMIT OF RESOLUTION}

In order to estimate the limit of resolution unambiguously, we follow a different approach that is based on the impulse (a)

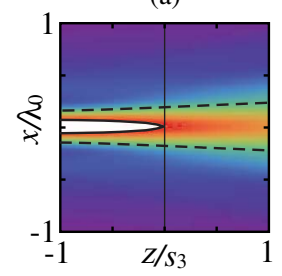

(d)

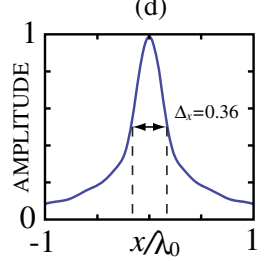

(b)

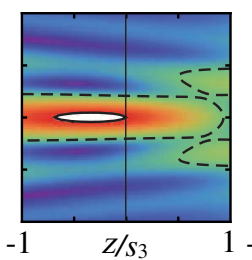

(c)

(f)
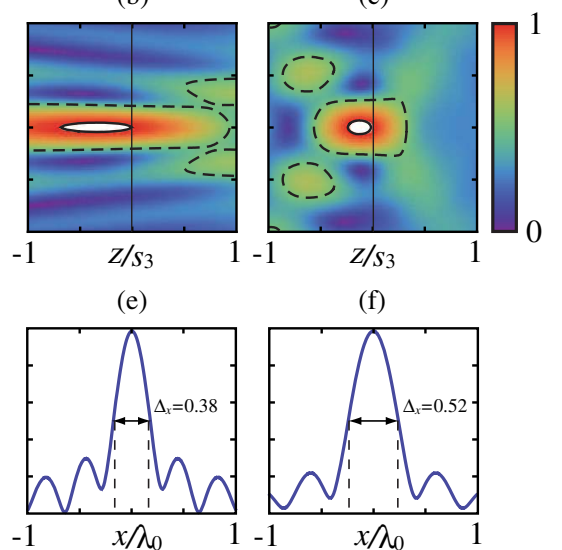

Fig. 7. (Color online) $|\vec{H}|$ from Eq. (15) as it is evaluated in the image space of a LHM flat lens with $\mu_{2}=-1+i 0.001$ and $\varepsilon_{2}=-4+i 0.001$ and different widths: (a) $d=0.125 \lambda_{0}$, (b) $d=0.875 \lambda_{0}$, and (c) $d=5.125 \lambda_{0}$. Again, the fields in the Gaussian image plane for (a), (b), and (c) are plotted in (d), (e), and (f), respectively. 
response of the optical system, also known as the PSF. This procedure may be carried out assuming that the imaging device under consideration is a linear and shift invariant (LSI) system. Note that a scattered field transmitted through a stack of dielectric-LHM layers remains unchanged in shape and magnitude if the scatterers are displaced in any direction that is parallel to the surfaces. Therefore the asymmetric LHM flat lens that we are considering is a LSI system in 2D. Moreover, the impulse response of the LHM superlens depends on the state of polarization of the wave field and, therefore, we may evaluate the PSF for $p$-polarized and $s$-polarized waves, independently. This is discussed thoroughly in [25], and here we only give a brief summary.

Based on the angular spectrum representation of the scattered field, for $s$-polarized waves, the electric field $\vec{E}$ in the image space may be expressed as a $2 \mathrm{D}$ convolution,

$$
\vec{E}\left(\vec{R}_{\perp}, z\right)=\vec{E}_{\mathrm{sc}}\left(\vec{R}_{\perp}\right) * h\left(\vec{R}_{\perp}, z\right),
$$

where $E_{\mathrm{sc}}$ is the wave field at the object plane and $\vec{R}_{\perp}=(x, y)$ stands for a space-domain $2 \mathrm{D}$ vector that is perpendicular to the unit vector $\hat{z}$. Strictly speaking, Eq. (16) is not restricted to scatterers that are located at the object plane. The scalar $3 \mathrm{D}$ $\mathrm{PSF}$ is

$$
h\left(\vec{R}_{\perp}, z\right)=\frac{1}{(2 \pi)^{2}} \iint t\left(\vec{k}_{\perp}\right) \exp \left(i \vec{k}_{\perp} \vec{R}_{\perp}+i \beta_{3} z\right) \mathrm{d} \vec{k}_{\perp}
$$

and is derived by using the transmission coefficient that corresponds to the object plane and its conjugate image plane as given in Eq. (7b). Note that $h\left(\vec{R}_{\perp}, z>0\right)$ for $t=1$ represents the propagator of the first Rayleigh-Sommerfeld integral, and it is related with a divergent wave whose focus is placed in the center of the image plane $z=0$ [26].

In fact, for symmetric flat lenses where $n_{1}=n_{3}$, Eq. (16) may be set as a $3 \mathrm{D}$ convolution by virtue of its property of shift invariance along the $z$-axis [25]. However, asymmetric flat lenses are not invariant under displacements on the optic axis. This is in agreement with our discussion in Section 2 concerning the correction of primary SA, which is achieved in a unique object plane.

In order to compare the PSF with the electric-field response of the LHM lens over a line electric dipole, calculated from Eq. (14), it is more appropriate to derive the PSF in 2D; that is,

$$
h_{2}(x, z)=\frac{1}{2 \pi} \int_{-\infty}^{\infty} t\left(k_{x}\right) \exp \left(i k_{x} x+i \beta_{3} z\right) \mathrm{d} k_{x} .
$$

In Fig. 8, we present the 2D PSF for the same superlens considered, for instance, in Fig. $\underline{6}$. One more time, the object plane is placed at a proper distance $s_{1}$ to compensate primary SA. We find again that the diffractive behavior of a slab width $d$ below the wavelength differs substantially from that lens with $d \gg \lambda_{0}$. Additionally, the impulse response is notably different for $s$-polarized waves and $p$-polarized waves. Note that the PSF for $p$-polarized waves is computed by using in Eq. (18) the corresponding coefficient of transmission $t$. The FWHM of the PSF central lobe for $s$-polarized waves takes higher values than those evaluated for $p$ polarization, especially in ultrathin LHM layers. For instance, if $d=0.125 \lambda_{0}$, (a)

(d)

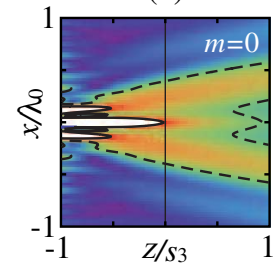

(g)

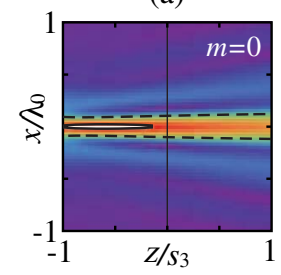

(b)

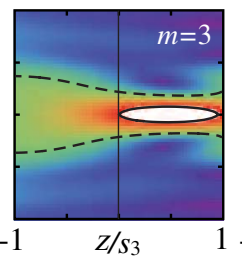

(e)

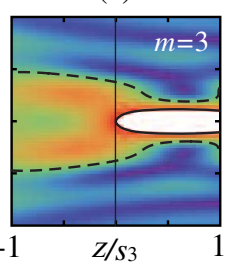

(h) (c)

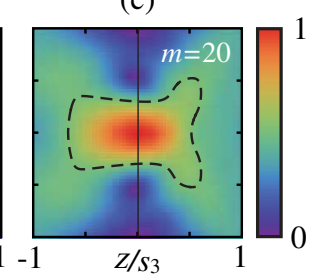

(f)

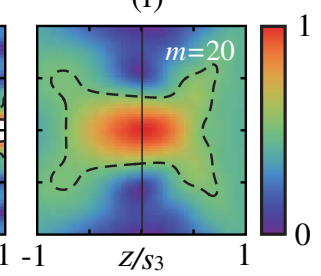

(i)

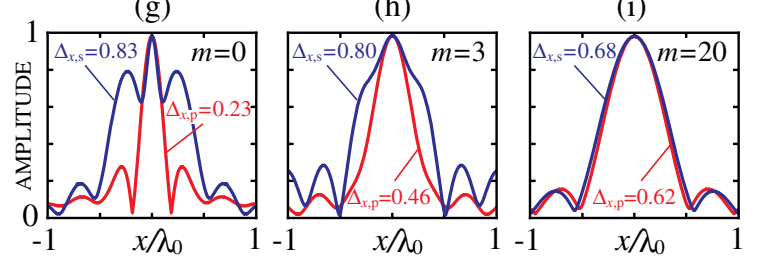

Fig. 8. (Color online) Modulus of the 2D PSF $\left|h_{2}\right|$ for a LHM flat lens with $\mu_{2}=-1+i 0.001$ and $\varepsilon_{2}=-4+i 0.001$ for different states of polarization: (a)-(c) applies for $p$-polarized waves and (d)-(f) for $s$ polarization. In (g)-(i), we chart the data for the Gaussian image plane. The slab width is also varied: $d=0.125 \lambda_{0}$ for subfigures placed in the left column, $d=0.875 \lambda_{0}$ for subfigures in the central column, and $d=5.125 \lambda_{0}$ for plots on the right.

shown in Fig. 8(g), the FWHM of the PSF in the Gaussian image plane yields $\Delta_{x, s}=0.83$ in units of $\lambda_{0}$, for $s$-polarized waves, which is much higher than the FWHM encountered for $p$-polarized waves, $\Delta_{x, p}=0.23$. However, differences derived by the state of polarization are negligible in the case $d=5.125 \lambda_{0}$, as shown in Fig. 8(i). From Figs. $\underline{8(\mathrm{c})}$ and 8(f), we conclude that this is true not only in the Gaussian image plane but also in out-of-focus planes.

Note that the FWHM from the PSF is slightly greater than that obtained in Section 4 from a line dipole. It is worthy to point out that a similar effect has been reported when comparing the PSF and the image of a subwavelength Gaussian beam in metal-dielectric multilayers [27]. In both cases, the explanation is nevertheless not difficult. The broader PSF has an irregular phase variation, not shown in Fig. 8, which is of critical relevance in the convolution given in Eq. (16). In a similar manner, fast changes in the phase of an incident wave field may lead to severe distortions in the image space. Obviously, phases of input fields and phases of PSFs would not play a role if the undulatory superposition (16) were fully incoherent. As a consequence, subwavelength signals transmitted by LHM coatings occasionally yield anomalous localized distributions whose FWHMs surpass the limit of resolution determined by the PSF.

\section{CONCLUSIONS}

We analyzed LHM superlenses in an asymmetric arrangement, focusing on primary aberrations and backscattering effects. Both are unwanted effects derived from impedance mismatch at boundaries. The IR of the LHM satisfying the antireflection coating condition $n_{2}=-\sqrt{n_{1} n_{3}}$ minimizes backscattered light. Provided that $d=(1+2 m) \lambda_{0} /\left(-4 n_{2}\right)$ and assuming that 
losses in the metamaterial are negligible, reflection of light at normal incidence is completely eliminated. To avoid primary SA, we show that the object plane will be placed at an appropriate distance from the front interface of the superlens. Nevertheless residual aberrations come out in the Gaussian conjugate plane, especially for slabs with a subwavelength width. Proximity of the source from the entrance surface of the lens and of the image from the exit interface, as it is compared with the wavelength, are crucial in order to exhibit a subwavelength resolution. Under these circumstances, coating superlenses may recover subwavelength information from the scattered wave field. Balancing residual aberrations may lead to focal shifts to achieve an impulse response of least spot size. Both finite-element analysis and PSF are used to estimate the limit of resolution for $s$-polarized and $p$-polarized waves.

\section{ACKNOWLEDGMENTS}

This research was funded by the Spanish Ministry of Economy and Competitiveness under the project TEC2009-11635.

\section{REFERENCES}

1. S. A. Ramakrishna, J. B. Pendry, D. Schurig, D. R. Smith, and S. Schultz, "The asymmetric lossy near-perfect lens," J. Mod. Opt. 49, 1747-1762 (2002).

2. P. Loschialpo, D. Forester, D. Smith, F. Rachford, and C. Monzon, "Optical properties of an ideal homogeneous causal left-handed material slab," Phys. Rev. E 70, 036605 (2004).

3. Z. Lin and Y. Zou, "Low-order aberration corrections of multilayer flat lenses using negative-index materials," Appl. Opt. 45, 6925-6931 (2006).

4. P. Valanju, R. Walser, and A. Valanju, "Wave refraction in negative-index media: always positive and very inhomogeneous," Phys. Rev. Lett. 88, 187401 (2002).

5. P. Loschialpo, D. Smith, D. Forester, F. Rachford, and J. Schelleng, "Electromagnetic waves focused by a negative-index planar lens," Phys. Rev. E 67, 025602 (2003).

6. T. Matsumoto, S. Fujita, and T. Baba, "Wavelength demultiplexer consisting of photonic crystal superprism and superlens," Opt. Express 13, 10768-10776 (2005).

7. T. Matsumoto, K.-S. Eom, and T. Baba, "Focusing of light by negative refraction in a photonic crystal slab superlens on silicon-on-insulator substrate," Opt. Lett. 31, 2786-2788 (2006).

8. T. Dumelow, J. da Costa, and V. Freire, "Slab lenses from simple anisotropic media," Phys. Rev. B 72, 235115 (2005).
9. H. Luo, Z. Ren, W. Shu, and F. Li, "Construction of a polarization insensitive lens from a quasi-isotropic metamaterial slab," Phys. Rev. E 75, 026601 (2007).

10. I. Bulu, H. Caglayan, and E. Ozbay, "Negative refraction and focusing of electromagnetic waves by metallodielectric photonic crystals," Phys. Rev. B 72, 045124 (2005).

11. J. Li and C. T. Chan, "Imaging using nano metallic films: from evanescent wave lens to resonant tunnelling lens," arXiv:physics/0701172v1 (2007).

12. T. Asatsuma and T. Baba, "Aberration reduction and unique light focusing in a photonic crystal negative refractive lens," Opt. Express 16, 8711-8719 (2008).

13. Q. Wu, J. M. Gibbons, and W. Park, "Graded negative index lens by photonic crystals," Opt. Express 16, 16941-16949 (2008).

14. D. Schurig and D. Smith, "Negative index lens aberrations," Phys. Rev. E 70, 065601 (2004).

15. J. Chen, C. Radu, and A. Puri, "Aberration-free negativerefractive-index lens," Appl. Phys. Lett. 88, 071119 (2006).

16. D. Schurig, J. B. Pendry, and D. R. Smith, "Transformationdesigned optical elements," Opt. Express 15，14772-14782 (2007).

17. V. G. Veselago, "The electrodynamics of substances with simultaneously negative values of $\epsilon$ and $\mu$," Physics-Uspekhi 10, 509-514 (1968).

18. V. N. Mahajan, Optical Imaging and Aberrations. Part I. Ray Geometrical Optics (SPIE, 1998).

19. N. Calander, "Surface plasmon-coupled emission and FabryPerot resonance in the sample layer: a theoretical approach," J. Phys. Chem. B 109, 13957-13963 (2005).

20. P. Yeh, Optical Waves in Layered Media (Wiley, 1988).

21. R. Marques, M. J. Freire, and J. D. Baena, "Theory of threedimensional subdiffraction imaging," Appl. Phys. Lett. 89, 211113 (2006).

22. B. Wood, J. B. Pendry, and D. P. Tsai, "Directed subwavelength imaging using a layered metal-dielectric system," Phys. Rev. B 74, 115116 (2006)

23. T. Hakkarainen, T. Setälä, and A. T. Friberg, "Subwavelength electromagnetic near-field imaging of point dipole with metamaterial nanoslab," J. Opt. Soc. Am. A 26, 2226-2234 (2009).

24. C. A. Balanis, Advanced Engineering Electromagnetics (Wiley, 1989).

25. C. J. Zapata-Rodríguez, D. Pastor, and J. J. Miret, "Threedimensional point spread function and generalized amplitude transfer function of near-field flat lenses," Appl. Opt. 49, 5870-5877 (2010).

26. M. Nieto-Vesperinas, "Problem of image superresolution with a negative-refractive-index slab," J. Opt. Soc. Am. A 21, 491-498 (2004)

27. R. Kotyński and T. Stefaniuk, "Multiscale analysis of subwavelength imaging with metaldielectric multilayers," Opt. Lett. 35, 1133-1135 (2010). 